\title{
NUMERICAL INVESTIGATION OF THE ACOUSTIC DAMPING OF PLANE ACOUSTIC WAVES BY PERFORATED LINERS WITH BIAS FLOW
}

\author{
DAN ZHAO AND ZHI YUAN ZHONG \\ School of Mechanics and Aerospace, Nanyang Technology University, 50 Nanyang Avenue \\ Singapore, 639798, Singapore \\ zhaodan@ntu.edu.sg,zzhong@ntu.edu.sg
}

\begin{abstract}
Perforated liners are extensively used in aero-engines and gas turbine combustors to suppress combustion instabilities. These liners, typically subjected to a low Mach number bias flow (a cooling flow through perforated holes), are fitted along the bounding walls of a combustor to convert acoustic energy into flow energy by generating vorticity at the rims of the perforated apertures. To investigate the acoustic damping of such liners with bias flow on plane acoustic waves, a time-domain numerical model is developed to compute acoustic wave propagation in a cylindrical duct with a single-layer liner attached. The damping mechanism of the liner is characterized in real-time by using a 'compliance', developed especially for this work. It is a rational function representation of the frequency-domain homogeneous compliance adapted from the Rayleigh conductivity of a single aperture with mean bias flow in the z-domain. The liner 'compliance' model is then incorporated into partial differential equations of the duct system, which are solved by using the method of lines. The numerical results are then evaluated by comparing with the numerical results of Eldredge and Dowling's frequency-domain model. Good agreement is observed. This confirms that the model and the approach developed are suitable for real-time characterizing the acoustic damping of perforated liners.
\end{abstract}

Keywords: Perforated liners; bias flow; homogeneous compliance; acoustic damping; plane waves.

\section{Introduction}

In order to meet more stringent requirements on emissions, combustors in ground based gas turbines and aero-engines need to operate under lean premixed conditions to achieve low $\mathrm{NO}_{\mathrm{x}}$. However, under lean premixed conditions, combustors are more susceptible to combustion instabilities. Combustion instabilities ${ }^{1}$ are generated by an interaction between acoustic waves and unsteady combustion. Unsteady heat release is an efficient acoustic source and generates acoustic waves. These pressure waves propagate within the combustor and partially reflect from boundaries to arrive back at the combustion zone, where they cause more unsteady heat release. Under certain conditions, this feedback can result in large and damaging self-excited oscillations.

To suppress combustion instabilities, the coupling between unsteady heat release and the pressure perturbation must somehow be interrupted. Passive controllers, such as perforated liners, are widely used as acoustic dampers to achieve this. Perforated liners are metal liners, frequently arranged in layers, which have tiny perforated holes in them ${ }^{2}$, ${ }^{3}$. In practice, a cooling air flow through the perforated holes (i.e. a bias flow) is needed 
to prevent the liners from being damaged by high temperature. Remarkably, the bias flow is found to increase liners damping performance. The main mechanism of acoustic damping involves vortex shedding generated over the rims of the perforated holes.

To study the liner's damping, Howe ${ }^{4}$ modeled the acoustic energy dissipated by the periodic shedding of vorticity for a single orifice in a high Reynolds-number flow using a Rayleigh conductivity. Following Howe's research, Hughes and Dowling ${ }^{5}$ studied the acoustic damping of screens with regular array of slits and circular perforations with mean bias flow, showing that all impinging sound could be absorbed at a particular frequency in theory. Jing and $\mathrm{Sun}^{3}$ experimentally investigated the effect of the screen thickness and the bias flow rate, showing that an appropriate bias flow rate can significantly increase damping and that the screen thickness is crucial. Recently Eldredge and Dowling ${ }^{2}$ performed both experimental and modeling work to investigate acoustic damping of a single or double-liner attached to a cylindrical duct. They used a 1D duct model with a homogeneous liner compliance to simulate a loudspeaker-driven cold-flow pipe system. It was found that there is an optimum bias flow rate around Mach number 0.2 , which can provide maximum acoustic damping. Mathematically, the corresponding time-domain compliance can be derived directly by taking the inverse Fourier transform of the frequency-domain compliance. However, this results in a convolution integral, which requires a long history of the bias flow fluctuation. Implementing the time-domain homogeneous compliance is impractical, especially for multi-dimensional problems.

Although majority of modeling work has been carried out in frequency domain, there are some attempts to simulate perforate liners' damping in time-domain. Sbardella et al. ${ }^{7}$ proposed a time-domain liner model, which combines a frequency-independent resistive part and a reactive part. Tam et al. ${ }^{8}$ carried out time-domain direct numerical simulation of a single liner and showed that vortex shedding is the dominant mechanism of absorption for incident waves of high amplitude. Reichert \& Biringen ${ }^{9}$ proposed a timedomain approach which introduced a source term to the momentum equations, and assessed the effect of a bias flow numerically. They found that with an optimum bias flow rate, the liners damping efficiency was significantly improved over that with no bias flow. Özyörük et al. ${ }^{10,11}$ use a z-transform method to convert the frequency-domain Myers $^{12}$ impedance boundary condition which accounts for the characteristics of the acoustic treated wall to a finite difference form, and therefore incorporate this impedance boundary condition into $1 \mathrm{D}$ and $2 \mathrm{D}$ Euler equations. They showed that applying $\mathrm{z}-$ transform technique can dramatically reduce the computation involved and so real-time simulations of liners damping is achieved.

The aim of this work is to develop a time-domain compliance based on the principle that unsteady vortex shedding from the perforated holes is the primary mechanism for acoustic wave dissipation. The liner's 'compliance' we proposed is formulated into a rational form in z-domain. And inverse z-transform of the compliance can provide an explicit expression describing the relationship between the fluctuating volume flux through the perforated holes and the fluctuating stagnation enthalpy across the holes. Thus with the mass continuity and the momentum equation along the duct, the 1D lined- 
duct model is developed. In Sec. 2, the model equations are developed and the ztransform method is introduced. In Sec. 3, numerical results are obtained for different flow conditions and compared with those from the model of Eldredge and Dowling ${ }^{2}$ in frequency-domain. To solve the partial differential equations, method of line (MOL) is adopted.

\section{Model}

\subsection{Liner equations}

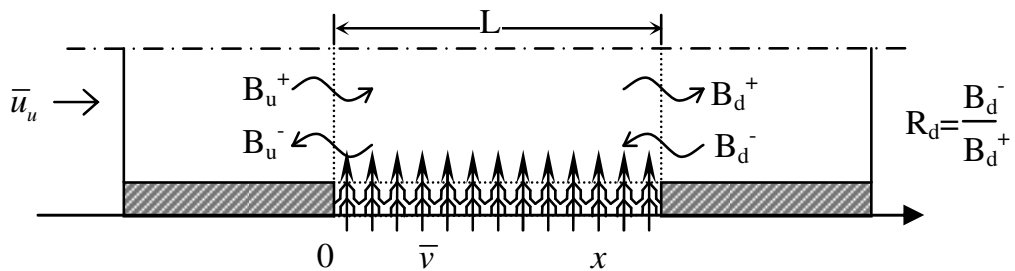

Fig. 1. Schematic of acoustic waves and flow in duct with bias-flow perforated liner.

In this work, we consider a configuration of a 1D cylindrical duct with uniform crosssectional area, consisting of a single-layer liner between two rigid wall sections, as shown in Fig. 1. The length of the liner is $L$ and the circumference and cross-sectional area is $C$ and $S$, respectively. An air flow with the velocity of $\bar{u}_{u}$ is present in the upstream duct, as well as a steady inward bias flow through the perforated holes of uniform average velocity of $\bar{v}$. Since both the axial and bias flow are small with the same stagnation temperature associated with them, the mean density and mean sound speed is uniform in the duct and denoted by $\bar{\rho}$ and $\bar{c}$, respectively. Then the axial mean flow velocity in the liner varying with $x$ along the liner can be expressed as:

$$
\bar{u}(x)=\bar{u}_{u}+\frac{C}{S} \bar{v} x .
$$

The equation of mass continuity over a control volume is:

$$
\frac{\partial \rho}{\partial t}+\frac{\partial}{\partial x}(\rho u)=\frac{C L}{S} \rho v
$$

Where $\rho, u, v$ is the instantaneous density, axial velocity and bias flow velocity, respectively. The term on the right hand side (RHS) of Eq. (2) is the source term introduced by the bias flow through the liner.

The equation of motion of the gas flow through the duct can be written as

$$
\rho \frac{\partial u}{\partial t}+\rho u \frac{\partial u}{\partial x}+\frac{\partial p}{\partial x}=0
$$


where $p$ is the instantaneous pressure. Consider the quantities in the above equation composed of mean and fluctuating part, for example, $\rho=\bar{\rho}+\rho^{\prime}$. Substituting $\rho, u, v$ in Eqs. (2) and (3) and linearizing them gives:

$$
\begin{gathered}
\frac{\partial \rho^{\prime}}{\partial t}+\bar{\rho} \frac{\partial u^{\prime}}{\partial x}+\bar{u} \frac{\partial \rho^{\prime}}{\partial x}=\frac{C}{S} \bar{\rho} v^{\prime}, \\
\bar{\rho} \frac{\partial u^{\prime}}{\partial t}+\bar{\rho} \bar{u} \frac{\partial u^{\prime}}{\partial x}+\frac{\partial p^{\prime}}{\partial x}=0 .
\end{gathered}
$$

Due to the presence of only plane waves and mean flow, it is more natural to describe the acoustic waves in terms of fluctuating stagnation enthalpy $B^{\prime}=p^{\prime} / \bar{\rho}+\bar{u} u^{\prime}$, where $p^{\prime}$ is the fluctuating pressure and $u^{\prime}$ is the fluctuating axial velocity. Thus the relationship between $\rho^{\prime}$ and $B^{\prime}$ holds as

$$
\rho^{\prime}=\frac{\bar{\rho} B^{\prime}}{\bar{c}^{2}}-\frac{\bar{\rho} \bar{u} u^{\prime}}{\bar{c}^{2}},
$$

where $B^{\prime}$ is the fluctuating enthalpy of the wave.

Substituting Eq. (6) into Eqs. (4) and (5) gives

$$
\begin{gathered}
\frac{\partial B^{\prime}}{\partial t}-\bar{u} \frac{\partial u^{\prime}}{\partial t}+\bar{c}^{2} \frac{\partial u^{\prime}}{\partial x}+\bar{u} \frac{\partial B^{\prime}}{\partial x}-\bar{u}^{2} \frac{\partial u^{\prime}}{\partial x}=\frac{C}{S} v^{\prime} \bar{c}^{2} \\
\frac{\partial u^{\prime}}{\partial t}+\frac{\partial B^{\prime}}{\partial x}=0 .
\end{gathered}
$$

Replacing $\partial u^{\prime} / \partial t$ in Eq. (7) with Eq. (8) leads to

$$
\frac{\partial B^{\prime}}{\partial t}+2 \bar{u} \frac{\partial B^{\prime}}{\partial x}+\left(\bar{c}^{2}-\bar{u}^{2}\right) \frac{\partial u^{\prime}}{\partial x}=\frac{C}{S} v^{\prime} \bar{c}^{2} .
$$

If two composite variables $\psi^{+}, \psi^{-}$are introduced, they are defined as

$$
\begin{aligned}
& \psi^{+}=\frac{1}{2 \bar{c}}(\bar{c}+\bar{u})\left[\left(B^{\prime}+(\bar{c}-\bar{u}) u^{\prime}\right],\right. \\
& \psi^{-}=\frac{1}{2 \bar{c}}(\bar{c}-\bar{u})\left[\left(B^{\prime}-(\bar{c}+\bar{u}) u^{\prime}\right] .\right.
\end{aligned}
$$

Their relations can be shown as $B^{\prime}=\psi^{+}+\psi^{-}$. After substituting Eqs. (10a) and (10b) into (9), two coupled linear partial differential equations can be derived, namely

$$
\begin{gathered}
\frac{\partial \psi^{+}}{\partial t}=-(\bar{c}+\bar{u}) \frac{\partial \psi^{+}}{\partial x}+\frac{C}{2 S} \bar{c}(\bar{c}+\bar{u}) v^{\prime}, \\
\frac{\partial \psi^{-}}{\partial t}=(\bar{c}-\bar{u}) \frac{\partial \psi^{-}}{\partial x}+\frac{C}{2 S} \bar{c}(\bar{c}-\bar{u}) v^{\prime}
\end{gathered}
$$


Similar equations are presented in Ref. 2. However, they are in a dimensionless form in frequency-domain.

The lined section boundaries are modeled using pressure reflection coefficients. The value of -0.98 is used at the open downstream end. This is close to the theoretical value of -1 for a negligible mean flow, but has been slightly decreased in magnitude to represent acoustic energy losses. This reflection coefficient $R_{d}$ relates the reflected wave to the incident wave at the boundary $x=L$, as

$$
\psi^{-}(t, L)=R_{d} \psi^{+}(t, L)
$$

At the upstream boundary of the lined section, we assume that the incident wave is known and it is given as

$$
\psi^{+}(t, 0)=B_{u}^{+}(t)
$$

If $v^{\prime}$ was known, then the time evolution of $\psi^{+}$and $\psi^{-}$could be determined. Because the bias flow fluctuation is related to the stagnation enthalpy inside the duct, an explicit expression describing the relationship is given as in Ref. 4 as

$$
\hat{v}=\frac{\eta}{i \omega}\left(-\hat{\psi}^{+}-\hat{\psi}^{-}\right)
$$

where $\omega$ is angular frequency, $i$ is the complex number and $\eta$ is liner compliance. It is given as

$$
\frac{1}{\eta}=\frac{\pi a^{2}}{\sigma} \frac{1}{K_{a}}+\frac{t}{\sigma},
$$

where $a$ and $t$ are the orifice radius and the thickness of the liner respectively, $\sigma$ is the open area ratio of the liner and $K_{a}$ is the Rayleigh conductivity as given by Howe ${ }^{3}$ as

$$
\begin{gathered}
K_{a}=2 a(\gamma+i \delta) \\
\gamma=\frac{I_{1}(S t)^{2}[1+1 / S t]+\left(4 / \pi^{2}\right) e^{2 S t} \cosh (S t) K_{1}(S t)^{2}[\cosh (S t)-\sinh (S t) / S t]}{I_{1}(S t)^{2}+\left(4 / \pi^{2}\right) e^{2 S t} \cosh ^{2}(S t) K_{1}(S t)^{2}} \\
\delta=\frac{(2 / \pi S t) I_{1}(S t) K_{1}(S t) e^{2 S t}}{I_{1}(S t)^{2}+\left(4 / \pi^{2}\right) e^{2 S t} \cosh ^{2}(S t) K_{1}(S t)^{2}}
\end{gathered}
$$

where $I_{1}$ and $K_{1}$ is modified Bessel functions and the Strouhal number, $S t=\omega a / U_{c}$. And $U_{c}$ is the mean convection velocity of vertical disturbances (approximated by $\bar{v} / \sigma$ ). Therefore, the compliance $\eta$ can be determined at specific mean bias flow velocity using Eqs. (15), (16) and (17).

It is obvious that the frequency-domain compliance can be converted into timedomain one by simply taking inverse Fourier transforms of Eq. (14). However, this will involve a convolution integral, and results in increased computation requirement. For multiple dimension problems, it is not practical to apply inverse Fourier transform to 
obtain the corresponding time-domain compliance. For this, we can propose a method to model the compliance in time-domain by using z-transform technique. It requires lees computation power and easily to be combine din the duct system equation. Detailed information can be found in the following section.

\subsection{Z-transform}

Following the work of Refs.10 and 11, the frequency-domain compliance could be first recast into z-domain since

$$
\frac{\partial}{\partial t}=i \omega=\frac{1-z^{-1}}{\Delta t}
$$

$\Delta t$ is the time step incensement for time integration. For simplicity we define a new compliance $Z=-\eta / \omega i$. Taking Z-transform at the both side of Eq. (14), we have

$$
v^{\prime}(z)=Z(z) B^{\prime}(z) \text {. }
$$

In general, the compliance $Z(z)$ can be modeled as

$$
Z(z)=\frac{a_{0}+\sum_{i=1}^{k} a_{i} z^{-i}}{1-\sum_{j=1}^{m} b_{j} z^{-j}},
$$

where $a$ 's and $b$ 's are constants which approximates $z$ accurately. For convergence and stability, the poles of the compliance should stay inside the unit circle. Substituting Eq. (20) into Eq. (19), taking the inverse z-transform and using the shift property, a time evolution description of $v^{\prime}$ can be shown namely

$$
v^{\prime(n)}=\sum_{j=1}^{m} b_{j} v^{\prime(n-j)}+\left(a_{0} B^{\prime(n)}+\sum_{i=1}^{k} a_{i} B^{\prime(n-i)}\right) .
$$

The superscript $n$ denotes the $n^{\text {th }}$ time step of the relevant quantities. Note that $B^{(n)}=\psi^{+(n)}+\psi^{-(n)}$.

\subsection{Modeling of the compliance in z-domain}

The forgoing shows that the frequency-domain compliance consists of both real and imaginary part. Thus the compliance needs to satisfy the following equation in frequency-domain

$$
Z(\omega)=\frac{\tilde{a}_{0}+\tilde{a}_{1} \omega i+\tilde{a}_{2} \omega^{2}+\tilde{a}_{3} \omega^{3} i+\tilde{a}_{4} \omega^{4}+\ldots}{\tilde{b}_{0}+\tilde{b}_{1} \omega^{2}+\tilde{b}_{2} \omega^{4}+\ldots},
$$

where $\tilde{a}$ and $\tilde{b}$ are constants used to estimate the values of compliance in the frequency range. Since the parameters $\tilde{a}$ and $\tilde{b}$ are determined, by substituting $\omega$ in the Eq. (18) 
into (21), the parameters of $a$ 's and $b$ 's can be easily indentified. In this paper a low-pass filter type of compliance is used as its model in frequency-domain

$$
Z(\omega)=\frac{1}{R_{0}+R_{1} \omega i} .
$$

The parameter $R_{0}$ and $R_{1}$ can be obtained through a nonlinear least square curve fit procedure based on Levenberg-Marquardt method ${ }^{13}$ according to the prediction of $Z(\omega)$ by using Eqs. (15)-(17). Fig. 2 shows the variation of the real and imaginary part of $Z(\omega)$ with frequency, when the mean flow through the pipe is $10.26 \mathrm{~m} / \mathrm{s}$.
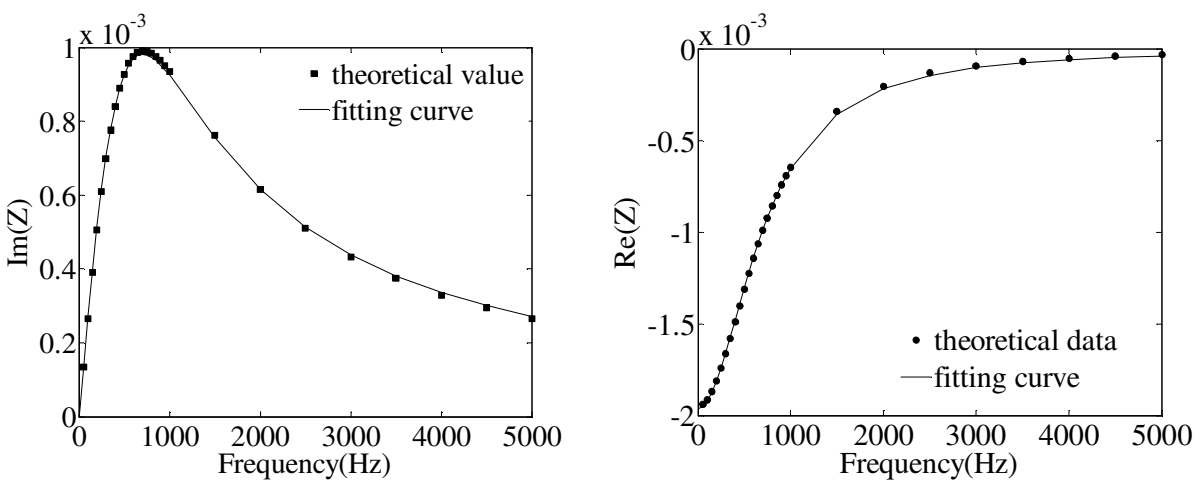

Fig. 2. The variation of the real and imaginary part of the compliance, $Z(\omega)=1 /\left(R_{0}+R_{1} \omega i\right)$ with frequency at $U_{c}=10.26 \mathrm{~m} / \mathrm{s}$.

The corresponding z-domain of the compliance can be easily shown as

$$
Z(z)=\frac{a_{0}}{1-b_{1} z^{-1}}
$$

where

$$
\begin{aligned}
& a_{0}=\Delta t /\left(\Delta t R_{0}+R_{1}\right), \\
& b_{0}=R_{1} /\left(\Delta t R_{0}+R_{1}\right) .
\end{aligned}
$$

\section{Numerical simulation}

\subsection{Discrete-time-domain model equations}

With the time-domain compliance, Eqs. (9) and (10) can be discretized and solved by converting them to ODEs by using the method of line ${ }^{14}$ (MOL). This technique has been widely used in solving PDEs discretizing in all but one dimension and leaving time variables continuous. To approximate the spatial derivatives, finite difference method is typically used. 
In our work, we discretize the spatial derivatives by using a three points, centered difference approximation and then obtain the semi-discrete form of Eqs. 11(a) and 11(b).

$$
\begin{aligned}
& \frac{\partial \psi_{i}^{+(n)}}{\partial t}=-(\bar{c}+\bar{u}) \frac{{\psi_{i+1}^{+}}^{(n-1)}-\psi_{i-1}^{+}{ }^{(n-1)}}{2 \Delta x}+\frac{C}{2 S} \bar{c}(\bar{c}+\bar{u}) \nu^{(n-1)}, \\
& \frac{\partial \psi_{i}^{-(n)}}{\partial t}=(\bar{c}-\bar{u}) \frac{\psi_{i+1}^{-}{ }^{(n-1)}-\psi_{i-1}^{-}{ }^{(n-1)}}{2 \Delta x}+\frac{C}{2 S} \bar{c}(\bar{c}-\bar{u}) \nu^{(n-1)}
\end{aligned}
$$

At the boundaries, i.e. $x=0$ and $x=L$

$$
\begin{aligned}
& \frac{\partial \psi_{1}^{(n-1)}}{\partial x}=\frac{-3 \psi_{1}^{(n-1)}+4 \psi_{2}^{(n-1)}-\psi_{3}^{(n-1)}}{2 \Delta x}, \\
& \frac{\partial \psi_{n}^{(n-1)}}{\partial x}=\frac{3 \psi_{n}^{(n-1)}-4 \psi_{n-1}^{(n-1)}+\psi_{n-2}^{(n-1)}}{2 \Delta x} .
\end{aligned}
$$

After substituting Eq. (24) into Eq. (19) and taking the inverse z-transform, a little algebra shows

$$
\frac{\partial v^{(n)}}{\partial t}=\frac{v^{(n)}-v^{(n-1)}}{\Delta t}=\frac{\left(b_{1}-1\right)}{\Delta t} v^{(n-1)}+\frac{a_{0}}{\Delta t} B^{(n)} .
$$

Eqs. (26) and (28) describing the 1D lined duct model can now be numerically solved by using a four-order Runge-Kutta scheme.

\subsection{Simulation results}

The acoustic damping of the perforated liners is characterized by calculating the acoustic wave energy being absorbed (power absorption coefficient). Power absorption is defined in terms of the incident and reflected waves on either side of the lined section as shown in Eq. (29).

$$
\Delta=1-\frac{\left|B_{d}^{+}\right|^{2}+\left|B_{u}^{-}\right|^{2}}{\left|B_{u}^{+}\right|^{2}+\left|B_{d}^{-}\right|^{2}},
$$

where $B_{u}^{+}, B_{d}^{+}, B_{d}^{-}$and $B_{u}^{-}$represent the upstream incident wave, the downstream incident wave, the downstream reflected wave and the upstream reflected wave. And, they are related to $\psi^{+}$and $\psi^{-}$in the form of

$$
\begin{aligned}
& \left|B_{u}^{+}\right|=\left|\psi^{+}(t, 0)\right|,\left|B_{d}^{+}\right|=\left|\psi^{+}(t, L)\right|, \\
& \left|B_{d}^{-}\right|=\left|\psi^{-}(t, L)\right|,\left|B_{u}^{-}\right|=\left|\psi^{-}(t, 0)\right| .
\end{aligned}
$$

The numerical results are shown in Figs. 3 and 4. It is shown in Fig. 3 that the variation of the power absorption with frequency at different bias flows. Comparisons are also made between our results and those of Eldredge and Dowling. To be consistent, we 
use $M_{u}=\bar{u}_{u} / \bar{c}$ and $M_{h}=U_{c} / \bar{c}$ to denote the Mach number of the pipe flow and the bias flow respectively. Figs. 3(a) and (b) illustrate the liner's damping performance in the absence of mean pipe flow. It is clear that with acoustic wave frequency increased, the liner's damping is dramatically increased. However, when the frequencies remain the same, but the bias flow Mach number is changed from 0.023 to 0.041 , the power absorption coefficient increase a little.

In the presence of the mean pipe flow, as shown in Figs. 3(c) and (d), the liners damping behaviors are different from the cases without mean pipe flow. When the bias flow Mach number is small, much less acoustic waves are being dissipated. However, with Mach number increased from 0.009 to 0.03 , the power absorption is increased by approximately $100 \%$ over the frequency range $400-700 \mathrm{~Hz}$. This might likely due to the more vortex shedding generated over the rims of the orifices over such frequency range.

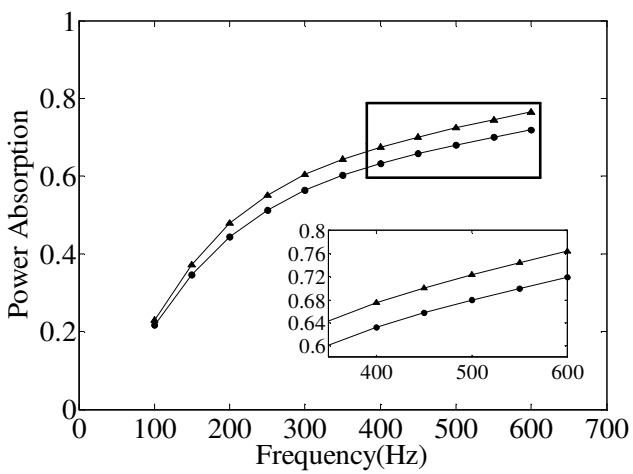

(a)

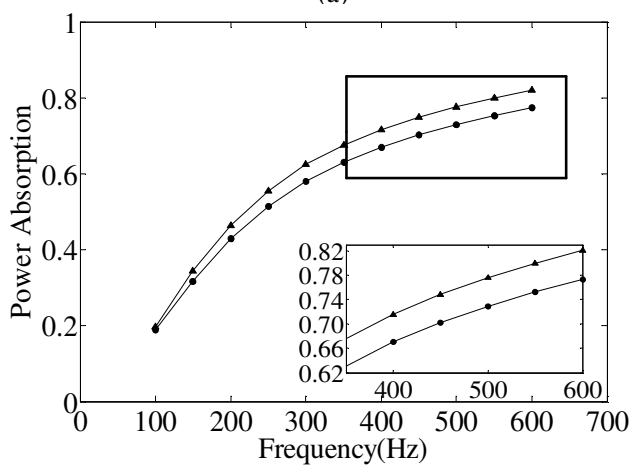

(c)

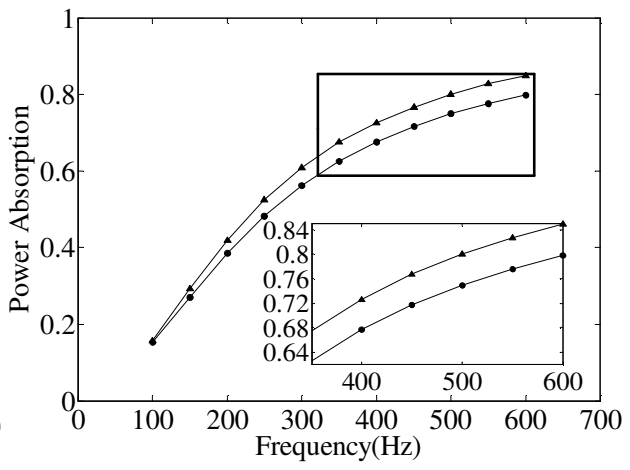

(b)

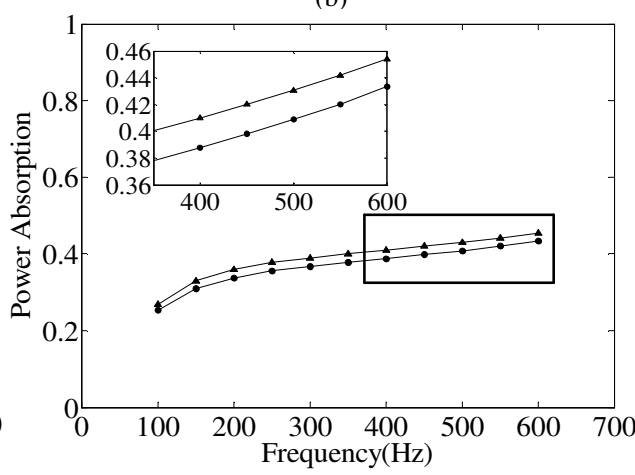

(d)

Fig. 3. Variation of power absorption with frequency for frequency for (a) $M_{h}=0.023, M_{u}=0$; (b) $M_{h}=0.041$, $M_{u}=0$; (c) $M_{h}=0.03, M_{u}=0.046$; (d) $M_{h}=0.009, M_{u}=0.057$. — - presenting model, $\_-\triangle$ Eldredge's results.

Fig. 4 shows the variation of the power absorption with bias flow Mach number at 4 different frequencies. The general trend for the power absorption is to increase with $M_{h}$ to a certain value and eventually decreases or maintain the same. This indicates that there is an optimum bias flow velocity at a given frequency. Close observation reveals that the 
maximum power absorption at the 4 different frequencies occurs at around $M_{h} \approx 0.03$. The optimum Mach number is insightful for supporting the idea of tuning liner's damping by optimizing the bias flow rate so that the liner's damping frequency range can be broadened. It can be seen from Fig. 4(d) that as high as $80 \%$ of incident waves will be dissipated at $600 \mathrm{~Hz}$. However, at lower frequency for example $272 \mathrm{~Hz}$, only $50 \%$ acoustic waves are absorbed. Comparing our results with those of Eldredge's model reveals that the power absorption is around 0.05 less. This could duo to the lower order compliance we developed. However, to verify our results, experiments needs to be performed. In addition, further investigation on higher order compliance is under process.
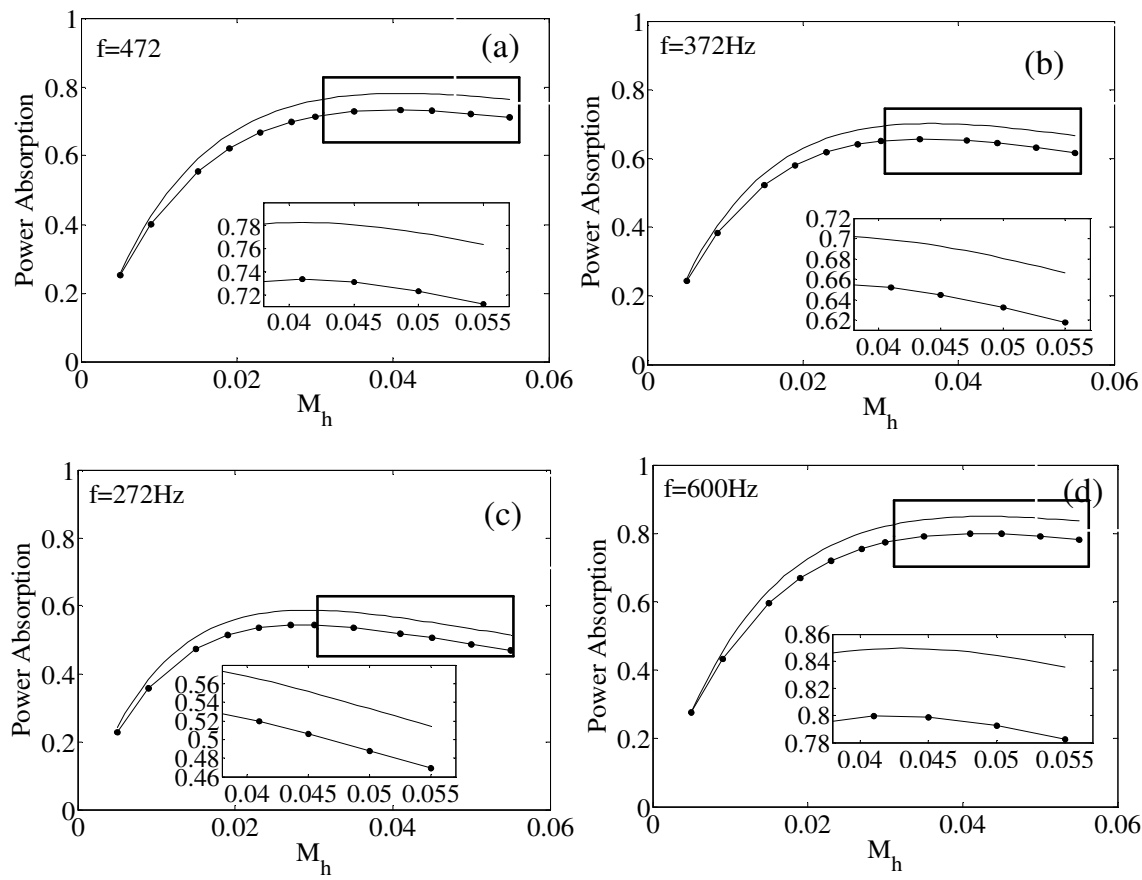

Fig. 4. Variation of power absorption with bias flow rate as at different frequencies: — - — presenting model, Eldredge's results.

\subsection{Modeling boundary condition with reflection coefficient}

The foregoing shows that the boundary condition at the downstream open end is described by using a reflection coefficient, which is assumed to be constant. However, this is not true for an unflanged open-ended pipe in practice. Levine and Schinger ${ }^{15}$ determined the reflection coefficient in the absence of mean duct flow. They used Wiener-Hopf approach to obtain the expression for the reflection coefficient as follow

$$
R_{d}=\frac{\varsigma\left(k R_{p}\right)-1}{\varsigma\left(k R_{p}\right)+1} \exp \left[-j 2 k\left(L+L_{d}\right)\right] .
$$


Where $\varsigma\left(k R_{n}\right)=0.25\left(k R_{n}\right)^{2}+j 0.6 k R_{n}$ is the specific acoustic impedance of the open end, $R_{n}$ is the radius of the duct, $k$ is the wave-number, $L$ and $L_{d}$ are the length of lined section and the downstream duct. Due to the vanishingly small Mach number under consideration, we use Eq. (31) to dynamically describe the reflection coefficient $R_{d}$. The liners damping is determined and shown in Fig. 5(a). It shows that the power absorption can be maximized to approximately $80 \%$. When the forcing frequency is lower $(<50 \mathrm{~Hz})$, negligible acoustic waves are being absorbed. However, as the forcing frequency is increased, the power absorption is increased dramatically and the maximum region becomes larger. It can also be seen that the liners damping depends on the acoustic wave frequency more strongly than on the bias flow rate at high frequencies. Compared with the case when $R_{d}=-0.98$ as shown in Fig. 5(b), it is obvious that the reflection coefficient plays an important role in the prediction of the acoustic damping of the liners. Therefore, the reflection coefficient needs to be properly modeled.

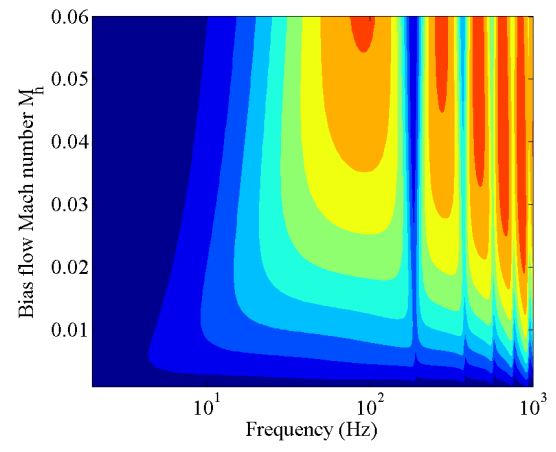

(a)

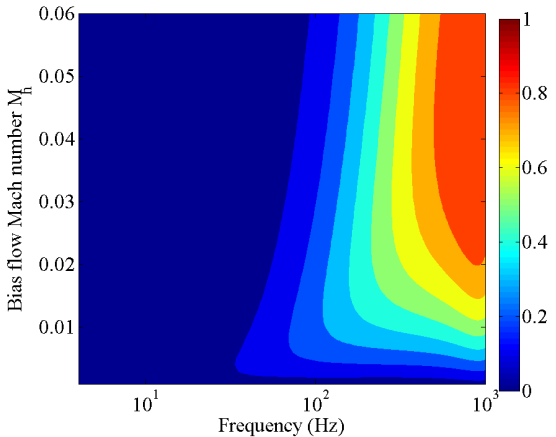

(b)

Fig. 5 Variation of power absorption with bias flow Mach number and frequency (a) $\mathrm{Rd}$ is calculated by Eq. (31), (b) $\mathrm{Rd}=-0.98$.

\section{Conclusions}

In this work, we proposed a time-domain compliance based on the principle that unsteady vortex shedding from the perforate holes is the primary mechanism for acoustic waves damping. The time-domain compliance is formulated in a rational function form by using z-transform technique. The compliance model is then incorporated into partial differential equations which describe 1D duct-lined model. Method of line is used to solve the system equations. The numerical results are evaluated by comparing with those of Eldredge and Dowling. Good agreement is observed. In addition, the influence of modeling the boundary conditions of the cylindrical duct on the liners damping is discussed. Our model is shown to be applicable to predict the liners' damping in realtime. 


\section{References}

1. T. C. Lieuwen and V. Yang, Combustion instabilities in gas turbine engines, (AIAA, Reston, 2005).

2. J. D. Eldredge and A. P. Dowling, J. Fluids Mech. 485, 307 (2003).

3. D. Zhao and A. S. Morgans, AIAA J. 49, 725 (2011).

4. M. S. Howe, Proc. R. Soc. Lond. A, 366, 205 (1979).

5. I. J. Hughes and A. P. Dowling, J. Fluids Mech. 218, 299 (1990).

6. X. Jing and X. Sun, J. Acoust. Soc. Am, 106, 2436 (1999).

7. L. Sbardella, B. J. Tester and M. Imregun, J. Sound Vib. 239, 379 (2001).

8. C. K. W. Tam, K. A. Kurbatskii, K. K. Ahja and R. J. Gaeta, J. Sound Vib. 245, 545 (2001).

9. R. S. Reichert and S. Biringen, Appl. Acoust. 62, 1049 (2001).

10. Y. Özyörük and L. N. Long, J. Comput. Acous. 5, 277 (1997).

11. Y. Özyörük and L. N. Long, J. Comput. Phys. 146, 29 (1998).

12. M. K. Myers, J. Sound Vib. 71, 429 (1980).

13. S. A. Teukolsky, W. T. Vetterling and B. P. Flannery, Numerical Recipes in Fortran, 2nd edn. (Cambridge University Press, New York, 1992).

14. W. E. Schiesser and G. W. Griffths, A Compendium of Partial Differential Equation Models (Cambridge University Press, New York, 2009).

15. H. Levine and J. Schwinger, Phys. Rev. 73, 383 (1948). 